\title{
Studies on modeling a single-bellows air spring and simulating its inherent characteristics
}

\author{
Yilong Zhang, Huirong Huang, Rongrong Chen, Minghui He \\ School of Mechanical and Electrical Engineering, Xi'an University of Architecture and Technology, \\ Xi'an 710055, China
}

Keywords: air spring, solid modeling, inherent characteristic, optimum structural design, finite element analysis.

\begin{abstract}
Pro-E and Ansys software are used for solid modeling of a single-bellows air spring, finite-element processing methods for this model are discussed, and the meshing usability is validated. Using Ansys software, we solve various orders of natural frequencies of a single-bellows air spring are solved at initial inflation pressures of 0.2, 0. 3 and 0. $4 \mathrm{MPa}$, Accordingly, the natural frequency of an air spring when operated can be regulated by adjusting its initial inflation, so as to make full use of air spring properties for engineering applications.
\end{abstract}

\section{Introduction}

The air spring is a new elastic vibration isolation element which is made in the rubber base material for a flexible sealed gas chamber filled with compressed air. It is a non-metallic spring using the compressibility of air to achieve resilient action. Because it has a low natural frequency, high frequency vibration absorption, noise performance, adjustable vertical stiffness and lateral stiffness and load capacity controlled by the internal pressure, etc., are widely used in many fields, such as launch vehicles, large aircraft, large ships and submarines and other important equipment, instrumentation aspects of vibration and noise reduction; land vehicles of locomotives, heavy trucks and limousines, are also extensive use of air spring vibration and noise reduction to improve ride comfort, typically vehicle suspension with air springs. This study focused on the use of a single bag of air spring Ansys software for modeling and simulation of its inherent characteristics.

\section{Single bag of air springs solid modeling and finite element treatment}

There are three kinds of air spring configuration mode: Air Spring, composite diaphragm air springs and air springs. Among them, the capsule can also be divided according to the air spring song number sac singles, hyperbolic, and three songs and other types. This study focused on the use of a single bag of air spring Ansys software for modeling and simulation of its inherent characteristics. Air spring finite element model can be divided into four parts: rubber balloon model, gas model, the model on the cover and the base model. Since the air spring is to analyze single capsule inherent characteristics, it is only considered in the initial filling pressure conditions, the vicinity of the equilibrium position, without gas model simulation. The cover model is a conventional mechanical seal fixing structure, and is mainly used and the bearing capacity of the base together with the rubber bladder seal. There is an air inlet, an air spring is used to charge and discharge the gas to the base. From one side of the rubber balloon can be seen ply cords be opened obliquely intersecting spread by vulcanization between two rubber layers, the density and the inclination of its cross-influencing material rigid air spring airbag. Its three-dimensional geometric model shown in Figure 1.The cord can be reduced to a rubber bladder in generalized plane stress state ax symmetric shell structure; it can be simplified into three-dimensional structural analysis of two-dimensional ax symmetric problem.

The model described above to deposit as a common format IGS imported into Ansys workbench model processing interface, two-dimensional model of the air spring after import in Figure 2. 


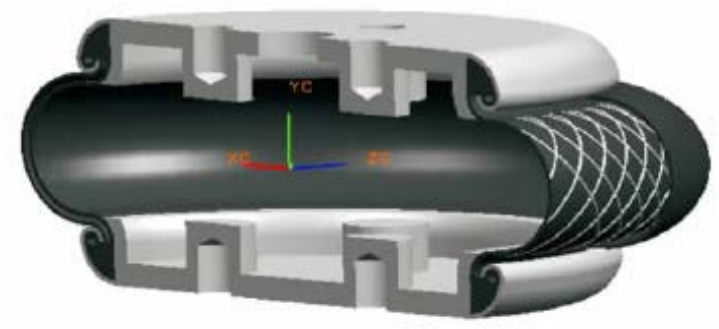

Fig.1 Layer diagram of the three-dimensional model of an air spring

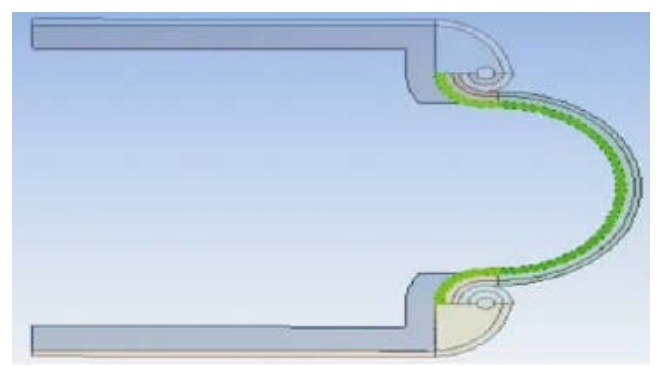

Fig.2 Two-dimensional model of an air spring after being imported into workbench

Model into Ansys workbench after considering the following the problem:

(1)For two-dimensional assembly model imported, must ensure that the law is consistent each plane direction, specifically by "DM-tools-surface lip" to change.

(2)In the air spring model material selection, its capsule base rubber material. Rubber materials are super elastic material, nearly incompressible, nonlinear characteristics. Unlike linear materials, rubber definition nonlinear curve fitting material by way of its constitutive models include Neo-Hookean, Mooney-Rivlin, Arruda-Boyce and Ogden, etc., which Mooney-Rivlin model cab be better describe the characteristics of the subject in the rubber air spring, it was adopted in this study.

(3) As the air spring is a combination of a rigid body and flexible body, combined with a variety of non-linear problems which, therefore exposure setting is very important.

(4) mesh is an important part of the finite element analysis, an important factor affecting the accuracy of the analysis and solution of scale, the division of the grid directly affect the quality of the model results accurate or not, because it is the plane model, so the use of triangle meshing grid type classification method for planar mapping method (mapped face meshing), it will give the mesh shape and approximate shape of a plane sheet, improve grid quality.

\section{Air spring inherent characteristic simulation}

Single-sac air spring natural frequency analysis of simulation results and analysis

Because it is the natural frequency of the air springs at different initial internal pressure and external loads without considering its impact, so the boundary conditions are not involved in external load loading conditions. In addition, only for Eigen value analysis initial equilibrium position; the same time as the rubber and the cover plate is always kept in contact, and no large relative sliding between the contact surfaces, the contact surface of the fixed connection processing. Thus solving linear. Ansys workbench using modal analysis module for the above models modal analysis.

Simulation operation specific boundary conditions are set as follows:

(1) Constraints. Need to add at one end of the air spring axially fix constraints, plus symmetry axis of symmetry constraints;

(2) Pressing need to define the initial conditions within the air spring in the air throughout the spring cavity, the modal analysis is the initial stress modal analysis using. In the initial inflation pressure of $0.2,0.3$ and $0.4 \mathrm{MPa}$ obtained when the natural frequency of the air spring shown in Figure 8, Figure4 and Figure 5. 


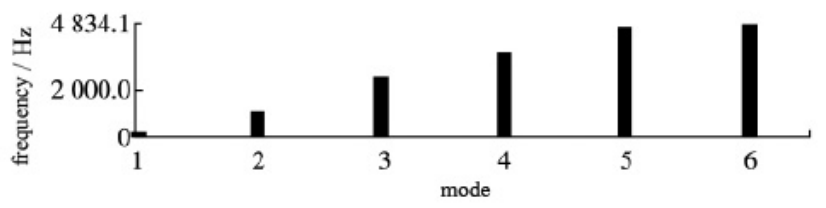

\begin{tabular}{cc}
\hline mode & frequency $/ \mathrm{Hz}$ \\
\hline 1 & 142.68 \\
2 & 992.61 \\
3 & 2506.20 \\
4 & 3630.60 \\
5 & 4701.30 \\
6 & 4834.10 \\
\hline
\end{tabular}

Fig.3 Air spring natural frequency values analyzed at an initial internal pressure of $0.2 \mathrm{MPa}$

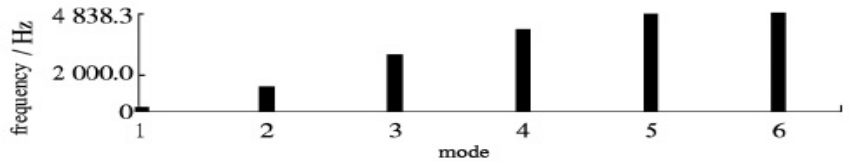

\begin{tabular}{cc}
\hline mode & frequency $/ \mathrm{Hz}$ \\
\hline 1 & 165.07 \\
2 & 1173.00 \\
3 & 2759.00 \\
4 & 3987.00 \\
5 & 4760.90 \\
6 & 4838.30 \\
\hline
\end{tabular}

Fig.4 Air spring natural frequency values analyzed at an initial internal pressure of $0.3 \mathrm{MPa}$

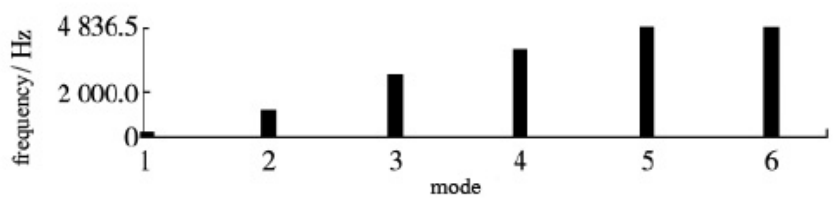

\begin{tabular}{cc}
\hline mode & frequency $/ \mathrm{Hz}$ \\
\hline 1 & 154.57 \\
2 & 1088.40 \\
3 & 2640.10 \\
4 & 3824.40 \\
5 & 4735.60 \\
6 & 4836.50 \\
\hline
\end{tabular}

Fig.5 Air spring natural frequency values analyzed at an initial internal pressure of $0.4 \mathrm{MPa}$

\section{Conclusion}

In this paper, By using the modeling software Pro-E and Ansys for single bagged air spring, discusses the finite element model of air spring processing method from assembly of the two-dimension , material selection, model of contact on the setting and mesh the model, and verify the availability of the grid division; By using the finite element model upside, carry out the simulation analysis of modal response for the single sac air spring under different initial inflation pressure, given the 1-4 step vibration shape diagram of the air spring under the internal pressure of $0.2 \mathrm{MP}$ and find out the weak points of the air spring model which is built, in order to improve the durability of the air spring need to optimize the structure of this part when designing.

\section{References}

[1] Liu Zenghua,Huang Yunhua. Control strategies for and emulation analysis on a semi-active air spring suspension system. Journal of System Simulation, 2007, 19(13) : 3022-3027.

[2] Yuan Chunyuan, Zhou Kongkang,Wu Linqi, et al.Structural analysis method of automotive air-spring rubber air-bag. Journal of Mechanical Engineering, 2009, 45(9) : 221-225.

[3] Cline C H O,False R. Solenoid damping of the pilot poppet in a forced-feedback metering poppet valve / / The ASME International Mechanical Engineering Congress and Exposition. Washington (USA) : ASMC, 2008: 409-414.

[4] Karts H,Van L L. Mitigation of high-frequency pulsations,using multi-hole restriction orifices / / The10th European Fluid Machinery Congress. Amsterdam (Netherlands) ,2008: 79-82.

[5] Yuan Chunyuan,Zhou Kongkang,Wu Linqi,et al. Finite element method to analyze vehicle air spring. Journal of Mechanical Engineering, 2009, 45(6) : 262-266. 
[6] Zhou Kongkang,Wu Linqi,An Dengfeng,et al. Finite element analysis for utricle's cord force of diaphragm air spring on vehicles. Journal of Jiangsu University Natural Science Edition, 2007, 28 (1): 21-24.

[7] James R S. Solution verification for explicit transient dynamics problems in the presence of hourglass and contact forces. Computer Methods in Applied Mechanics and Engineering, 2006, 195(13 /14 /15 /16): 1499-1516.

[8] Karts H,Van L L. Mitigation of high-frequency pulsations, using multi-hole restriction orifices / / The10th European Fluid Machinery Congress. Amsterdam (Netherlands) ,2008: 79-82. 